\title{
Single-use suvorexant for treating insomnia during overnight polysomnography in patients with suspected obstructive sleep apnea: a single-center experience
}

This article was published in the following Dove Medical Press journal:

Drug Design, Development and Therapy

\author{
Takuma Matsumura' \\ Jiro Terada' \\ Chikara Yoshimura ${ }^{2}$ \\ Ken Koshikawa' \\ Taku Kinoshita' \\ Misuzu Yahaba' \\ Kengo Nagashima ${ }^{3}$ \\ Seiichiro Sakao' \\ Koichiro Tatsumi' \\ 'Department of Respirology, \\ Graduate School of Medicine, Chiba \\ University, Chiba, Japan; ${ }^{2}$ Department \\ of Respiratory and Sleep Medicine, \\ Fukuoka University School of \\ Medicine, Fukuoka, Japan; ${ }^{3}$ Research \\ Center for Medical and Health Data \\ Science, The Institute of Statistical \\ Mathematics, Tokyo, Japan
}

Purpose: Although patients with suspected obstructive sleep apnea (OSA) might suffer difficulty in falling asleep during overnight polysomnography (PSG), standard hypnotics to obtain sleep during PSG have not been established. The aim of this study was to investigate the safety and efficacy of a new hypnotic agent, suvorexant, a dual orexin receptor antagonist, for insomnia in suspected OSA patients during in-laboratory PSG.

Patients and methods: An observational study was conducted during PSG for 149 patients with suspected OSA who had no insomnia at home. Patients with difficulty in falling asleep during PSG were optionally permitted to take single-use suvorexant. Patients with residual severe insomnia ( $>1$ hour) after taking suvorexant were permitted to take an add-on use zolpidem. Clinical data and sleep questionnaire results were analyzed between a no insomnia group (without hypnotics) and an insomnia group (treated with suvorexant).

Results: Among 84 patients who experienced insomnia during PSG and required hypnotics (the insomnia group; treated with suvorexant), 44 (52.4\%) achieved sufficient subjective sleep with single-use of suvorexant, while the other $40(47.6 \%)$ required suvorexant plus zolpidem. An apnea hypopnea index (AHI) of $\geq 5$ was observed in 144 out of 149 patients with predominantly obstructive respiratory events. Among those patients, $70.8 \%$ in the no insomnia group and $63.1 \%$ in the insomnia group had severe OSA. Regarding both subjective sleep time and morning mood, significant differences between the no insomnia group and the insomnia group were not observed. No patient taking suvorexant had an adverse event, such as delirium or falling. Conclusion: Single-use suvorexant seems to be a safe and effective (but mild) hypnotic agent for suspected OSA patients with insomnia during in-laboratory PSG.

Keywords: insomnia, suvorexant, polysomnography, obstructive sleep apnea, zolpidem, natural sleep

\section{Introduction}

In-laboratory overnight polysomnography (PSG) has been still widely conducted as a gold standard test to diagnose obstructive sleep apnea (OSA). Although most patients with suspected OSA can undergo PSG with substantial sleep due to the symptom of severe sleepiness, some patients suffer difficulty in falling asleep during PSG. This insomnia is partly explained by a "first-night effect" of PSG recordings, a well-known phenomenon caused by discomfort with the PSG electrode cables, limitation of movement, and/or maladaptation to the unfamiliar circumstances of a sleep laboratory, ${ }^{1,2}$ especially in anxiety disorders patients and older patients. ${ }^{3,4}$ Because the first-night
Correspondence: Jiro Terada Department of Respirology, Graduate School of Medicine, Chiba University, I-8-I Inohana, chuo-ku, Chiba city, Chiba, Japan

Tel $+8|432227| 7 \mid$

Fax +81432262176

Email jirotera@chiba-u.jp 
effect causes a decrease in total sleep time (TST), frequent nocturnal awakening, and disruption of sleep structure (eg, increased stage N1 sleep, reduced rapid eye movement [REM] sleep, and longer REM latency), ${ }^{5}$ more adequate amount of natural sleep during the sleep test is preferred. However, conducting PSG on multiple nights seems impractical due to the cost benefit, patient burden, and limited resources. Additionally, the use of an ideal hypnotic agent to mitigate insomnia during PSG has not been standardized.

Benzodiazepines and/or non-benzodiazepines (ie, type A gamma-aminobutyric acid $\left[\mathrm{GABA}_{\mathrm{A}}\right]$ receptor hypnotic agents) are most frequently used to treat insomnia worldwide, even during hospitalization. ${ }^{6}$ However, there are several concerns about the routine use of $\mathrm{GABA}_{\mathrm{A}}$ receptor agonists for patients with insomnia who might have undiagnosed OSA. First, benzodiazepines have the potential to decrease upper airway muscle activity and ventilatory responses to carbon dioxide, which could confound the disease state of sleep apnea. ${ }^{7-9}$ Although both benzodiazepines and non-benzodiazepines did not increase apnea index in patients with mild-to-moderate OSA, ${ }^{10,11}$ the effects of $\mathrm{GABA}_{\mathrm{A}}$ receptor hypnotic agents in patients with severe OSA is yet unknown. Second, GABA receptor hypnotic agents can be occasionally associated with delirium and falling, which may cause bone fractures, especially in elderly patients. ${ }^{12-14}$ Third, benzodiazepines are known to disrupt natural sleep architecture by decreasing slow wave sleep and REM sleep, and non-benzodiazepines are controversially reported to reduce REM sleep. ${ }^{15,16}$

In 2014, the US Food and Drug Administration (FDA) approved suvorexant (Merck \& Co., Inc., Whitehouse Station, NJ, USA), a new class of hypnotic agent, which blocks both orexin receptor type 1 and 2, for the treatment of insomnia with sleep onset and/or sleep maintenance difficulties. Since orexin is a neuropeptide that promotes wakefulness and affects the sleep-wake cycle, ${ }^{17,18}$ this orexin receptor antagonist can represent a novel approach to treat insomnia; current benzodiazepine receptor agonists appear to promote sleep by increasing the function of GABA, the major inhibitory neurotransmitter in the whole brain. Therefore, the new hypnotic agent, suvorexant, is expected 1) to have limited effects on sleep architecture and EEG power spectrum (ie, preserve natural sleep), ${ }^{19,20}$ 2) to lack clinically important respiratory effects during sleep, ${ }^{21}$ and 3 ) to have less serious adverse effects compared with $\mathrm{GABA}_{\mathrm{A}}$ receptor hypnotic agents, because it has no effect on the $\mathrm{GABA}_{\mathrm{A}}$ receptor. ${ }^{22}$ Accordingly, suvorexant might have potential as a useful hypnotic agent for patients with insomnia during overnight PSG. However, no study has yet investigated the efficacy and safety of single-use suvorexant for patients with all severities of OSA during PSG. In this study, we investigated the efficacy and safety of suvorexant in suspected OSA patients with insomnia during in-laboratory PSG in a single center.

\section{Materials and methods Ethical approval}

All study procedures involving human participants were approved by the Ethical Review Board of the Graduate School of Medicine of Chiba University (approval number 2584). The study protocol was conducted in accordance with the ethical principles of 1964 Helsinki Declaration and subsequent amendments. All patients provided written informed consent before undergoing the tests and procedures prior to the PSG recording.

\section{Subjects and study design}

This research is an observational retrospective study which included 190 consecutive patients who underwent in-laboratory PSG with clinically suspected OSA in Chiba University Hospital from April 2016 to March 2018. Patients who used hypnotics at home, those who were younger than 18 years old, and those who underwent deep brain stimulation surgery were excluded (Figure 1).

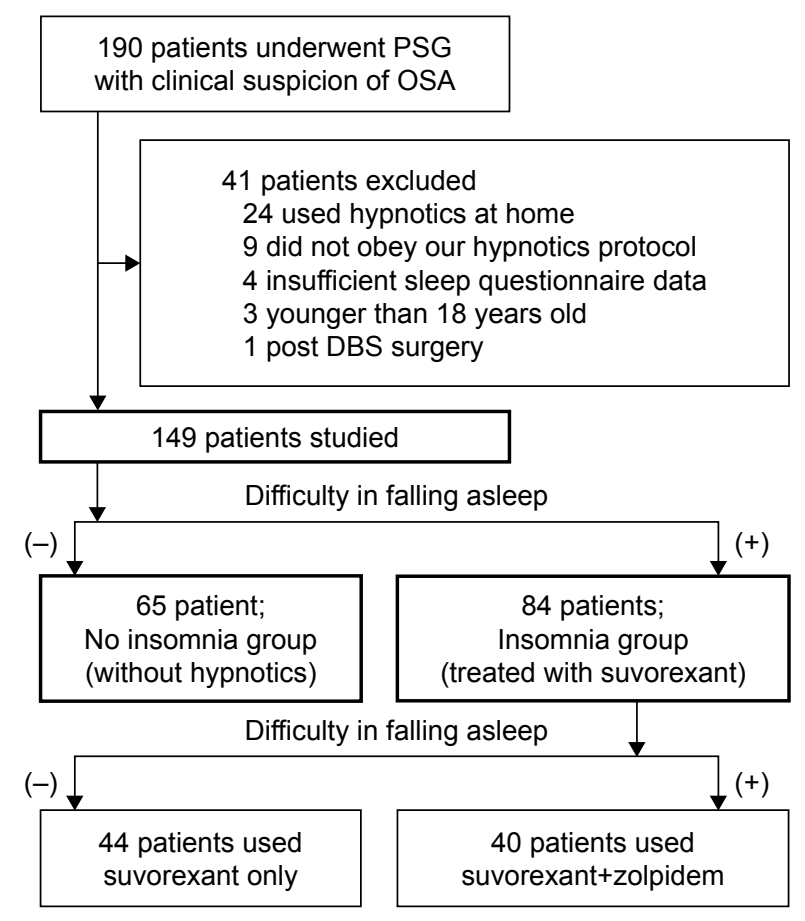

Figure I Flowchart of patient selection.

Notes: Of 190 patients who were screened, 65 were assigned to the no insomnia group (without hypnotics) and 84 to the insomnia group (treated with suvorexant). In the insomnia group, 40 patients required zolpidem after suvorexant. $(+)=$ Yes, $(-)=$ No. Abbreviations: DBS, deep brain stimulation; OSA, obstructive sleep apnea; PSG, polysomnography. 
In principle, the lights were turned off in the rooms at 9:00 pm, and all patients prepared to sleep at that time. Patients with difficulty in falling asleep were first optionally permitted to take single-use suvorexant before 12:00 am. Patients with residual severe difficulty in falling asleep 1 hour after taking suvorexant were additionally permitted to take add-on use zolpidem $(5 \mathrm{mg})$. The dose of suvorexant was decided as $15 \mathrm{mg}$ in this study according to a phase III trial including 247 Japanese (P028 trial), in which the clinical dose of suvorexant was decided as $20 \mathrm{mg}$ for adult patients and $15 \mathrm{mg}$ for elderly patients in Japan. ${ }^{23}$

Patients were analyzed in two groups according to hypnotic usage: a no insomnia group of patients who did not use hypnotics during PSG, and an insomnia group of patients who used suvorexant during PSG. Patients in the insomnia group were also divided into two groups according to zolpidem usage: a suvorexant only group of patients, who used only suvorexant during PSG, and a suvorexant+zolpidem group of patients, who used suvorexant plus additional zolpidem during PSG. Patients who did not obey our hypnotics protocol were excluded (Figure 1).

\section{PSG recording and scoring}

PSG was scored according to the American Academy of Sleep Medicine Manual for the Scoring of Sleep and Associated Events, version 2.3. Apnea was defined as a reduction in nasal airflow to $<10 \%$ of baseline for 10 seconds or longer, while hypopnea was defined as a reduction in nasal airflow signal amplitude of $\geq 30 \%$ for 10 seconds or longer in association with either a $\geq 3 \%$ oxygen desaturation or electroencephalographic arousal. OSA was defined as an apnea hypopnea index (AHI) of five or more events per hour combined with predominantly obstructive respiratory events. OSA severity was classified according to AHI values as mild, 5-15; moderate, $>15-30$; or severe, $>30$. Periodic leg movements were scored according to the World Association of Sleep Medicine criteria. ${ }^{24}$ According to the International Classification of Sleep Disorders, third (ICSD-3) criteria, periodic limb movements during sleep (PLMS) index $>15$ was considered abnormal.

\section{Clinical data and sleep questionnaire}

Clinical patient characteristics, including age, sex, body mass index, Epworth sleepiness scale score, usage of psychotropic drugs at home, and usage of hypnotics during PSG were collected from clinical records. Data analyses were consecutively conducted upon admission of each patient to the Sleep Disordered Breathing Clinic of Chiba University Hospital.
Patients were asked to answer an ordinary sleep questionnaire in the morning after PSG. The sleep questionnaire consisted of two questions regarding a) subjective sleep time compared with home ("Longer," "No change," or "Shorter") and b) morning mood compared with home ("Better," "No change," or "Worse"). Patients with insufficient sleep questionnaire data were excluded (Figure 1).

\section{Statistical analyses}

All statistical analyses were performed using JMP Pro 13.2.1 software (SAS Institute Inc., Cary, NC, USA), and all data are expressed as medians (interquartile ranges). The MannWhitney U-test was used to determine the significance of differences and Fisher's exact test was used to determine differences in the proportions of groups. All tests were twotailed and a probability $(P)$ value of $<0.05$ was considered statistically significant.

\section{Results}

\section{Patient characteristics}

The clinical characteristics of patients are shown in Table 1. One hundred and ninety patients were assessed for eligibility. After excluding 41 patients, 149 patients (65 patients in the no insomnia group [without hypnotics] and 84 patients in the insomnia group [treated with suvorexant]) were studied (Figure 1). In the insomnia group, 44 (52.4\%) of 84 patients had substantial subjective sleep with single-use suvorexant, and the other $40(47.6 \%)$ required additional zolpidem due to residual subjective difficulty in falling asleep after taking suvorexant. The majority of patients in both groups were middle-aged obese males. Subjective daytime sleepiness was deemed not severe, the median Epworth sleepiness scale score of all patients was $8.0($ range $=5.0-12.0)$. Some patients used psychotropic drugs at home. There were no significant differences in patient characteristics between the two groups, except antidepressant usage.

\section{PSG data}

PSG data are shown in Table 2. Sleep efficiency was significantly lower in the insomnia group and relatively low in both groups. Since patients with severe insomnia with sleep onset took suvorexant (Figure 1), sleep latency was significantly longer in the insomnia group, as compared with the no insomnia group. However, there was no significant difference in TST between the two groups. The median rates of \%Stage N3 in the no insomnia group and the insomnia group were $2.5 \%$ and $3.2 \%$, respectively, suggesting poor sleep architecture in both groups. An AHI of $\geq 5$ was 
Table I Patient characteristics

\begin{tabular}{l|l|l|l}
\hline & No insomnia group $(\mathbf{n}=\mathbf{6 5})$ & Insomnia group $(\mathbf{n}=\mathbf{8 4})$ & $\boldsymbol{P}$-value \\
\hline Age (years) & $54.0(44.0-66.0)$ & $60.0(51.0-69.0)$ & 0.055 \\
Male sex & $49(75.4)$ & $60(71.4)$ & 0.71 \\
Body mass index $\left(\mathrm{kg} / \mathrm{m}^{2}\right)$ & $27.7(23.8-30.1)$ & $26.5(23.4-30.2)$ & 0.59 \\
Epworth sleepiness scale & $7.0(5.0-12.0)$ & $8.0(5.0-12.0)$ & 0.71 \\
Psychotropic drugs & $7(10.8)$ & $5(6.0)$ & 0.37 \\
$\quad$ Antipsychotics & $I(1.5)$ & $2(2.4)$ & 1.00 \\
Antidepressant & $5(7.7)$ & $0(0.0)$ & $0.014^{*}$ \\
Antianxiety & $5(7.7)$ & $3(3.6)$ & 0.30 \\
\hline
\end{tabular}

Notes: Data are presented as the median (interquartile range) for numerical data and $\mathrm{n}$ (\%) for categorical data. $* \mathrm{P}<0.05$.

observed in 144 out of 149 patients, and all displayed predominantly obstructive respiratory events. Severe sleep apnea (AHIAHI $>30$ ) occurred in $46 / 65$ patients $(70.8 \%$ ) in the no insomnia group and 53/84 patients (63.1\%) in the insomnia group. The severity of sleep apnea, as determined by AHI, oxygen desaturation index (ODI), and \% time spent with $\mathrm{SpO}_{2}<90 \%\left(\% \mathrm{SpO}_{2}<90 \%\right)$, were not significantly different between the two groups. AHI, ODI, or $\% \mathrm{SpO}_{2}<90 \%$ did not differ significantly between the suvorexant only group and the suvorexant+zolpidem group, neither (data not shown). A PLMS index greater than 15 was noted in $9.2 \%$ of patients in the no insomnia group and $14.3 \%$ of those in the insomnia group, without significant difference.

\section{Sleep questionnaire}

The questionnaire results related to subjective sleep symptoms are shown in Figure 2 and Figure S1. The majority of the patients answered that sleep time was longer or no change and morning mood was better or no change compared to those at home in all the groups. There were no significant differences between the no insomnia group and the insomnia group and between the suvorexant only group and the suvorexant+zolpidem group regarding both subjective sleep time and morning mood. The subjective sleep latency was almost consistent with sleep latency detected by PSG (Figure S1 and Table 2).

\section{Adverse events}

No patient fell and experienced delirium during the study period in either group.

\section{Discussion}

In this study, several important features regarding single-use suvorexant for insomnia in suspected OSA (all: 149 patients, OSA: 144/149 patients, severe OSA: 99/144 patients)

Table 2 Summary of PSG data

\begin{tabular}{|c|c|c|c|}
\hline & No insomnia group $(n=65)$ & Insomnia group $(n=84)$ & $P$-value \\
\hline TST (min) & $384.0(327.5-461.3)$ & $356.8(315.6-423.8)$ & 0.062 \\
\hline Sleep efficiency (\%) & $66.6(57.3-81.8)$ & $62.9(52.9-73.1)$ & $0.043^{*}$ \\
\hline Sleep latency (min) & $28.1(14.0-50.9)$ & $46.1(24.1-78.9)$ & $0.010 *$ \\
\hline REM latency (min) & $105.0(75.8-165.3)$ & $138.8(97.3-191.1)$ & 0.084 \\
\hline Stage NI (\% TST) & $25.8(|6.5-4| . \mid)$ & $25.0(\mid 3.6-36.1)$ & 0.23 \\
\hline Stage N2 (\% TST) & $48.7(35.7-57.8)$ & $47.8(38.9-55.1)$ & 0.79 \\
\hline Stage N3 (\% TST) & $2.5(0.0-11.3)$ & $3.2(0.0-13.7)$ & 0.38 \\
\hline REM (\% TST) & $17.6(12.3-24.3)$ & $19.0(14.3-23.2)$ & 0.43 \\
\hline WASO & $144.5(92.8-212.8)$ & $162.5(112.5-203.8)$ & 0.36 \\
\hline Arousal index (events/h) & $40.5(28.5-54.9)$ & $37.1(27.6-62.3)$ & 0.80 \\
\hline $\mathrm{AHI}$ (events/h) & $39.7(28.9-58.0)$ & $37.4(21.6-60.5)$ & 0.72 \\
\hline $3 \%$ ODI & $31.4(19.8-46.1)$ & $27.5(16.5-40.5)$ & 0.31 \\
\hline $4 \%$ ODI & $22.3(11.5-40.2)$ & $19.7(9.4-34.1)$ & 0.64 \\
\hline$\% \mathrm{SpO}_{2}<90 \%$ & $3.2(0.4-8.2)$ & $4.1(0.7-11.8)$ & 0.31 \\
\hline Mean $\mathrm{SpO}_{2}(\%)$ & $95.0(94.0-96.0)$ & $95.0(94.0-97.0)$ & 0.95 \\
\hline Lowest $\mathrm{SpO}_{2}(\%)$ & $77.0(71.0-85.0)$ & $76.5(71.0-84.8)$ & 0.78 \\
\hline PLMS index (events/h) & $0.0(0.0-3.0)$ & $0.0(0.0-2.3)$ & 0.57 \\
\hline
\end{tabular}

Notes: Data are presented as the median (interquartile range). $* P<0.05$

Abbreviations: AHI, apnea-hypopnea index; ODI, oxygen desaturation index; PLMS, periodic limb movements during sleep; PSG, polysomnography; REM, rapid eye movement; $\% \mathrm{SpO}_{2}<90 \%$, \% time spent with $\mathrm{SpO}_{2}<90 \%$; TST, total sleep time; WASO, wake time after sleep onset. 

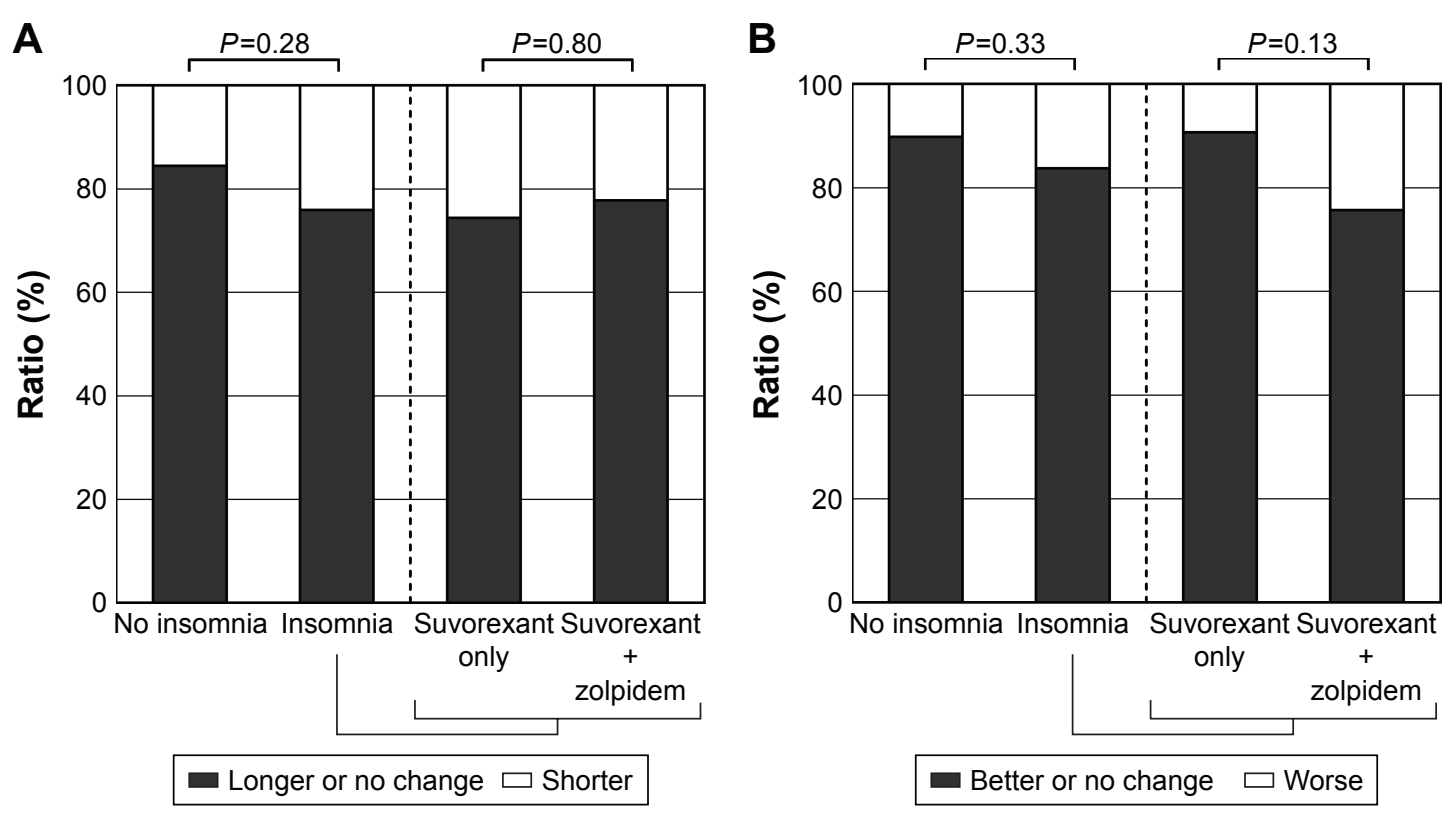

Figure 2 Summary of sleep questionnaire responses: subjective sleep time compared with home (A), and morning mood compared with home (B).

Notes: The majority of the patients answered the subjective sleep time was longer or no change and morning mood was better or no change compared to those at home in all the groups. There were no significant differences between the no insomnia group and the insomnia group and between the suvorexant only group and the suvorexant+zolpidem group regarding both subjective sleep time and morning mood.

patients during overnight PSG were found. First, 84 (56.4\%) of 149 patients with OSA who originally had no insomnia at home experienced difficulty in falling asleep during PSG and required the use of one or two hypnotic agents to sleep. Among these 84 patients, 44 (52.4\%) achieved sufficient sleep with single-use suvorexant, while the other $40(47.6 \%)$ required suvorexant plus zolpidem. Second, both subjective sleep time and morning mood in the majority of patients in the insomnia group were not different from those at home. Additionally, 84 patients who used suvorexant had no apparent adverse event, such as delirium or falling.

In the present study, suvorexant had some substantial effects for patients with difficulty in falling asleep during in-laboratory PSG, although GABA ${ }_{A}$ receptor agonists had been conventionally used to treat insomnia in our facility. Approximately half of the patients with suvorexant administration needed add-on hypnotics to sleep during overnight PSG; therefore, the effect of single-use suvorexant on sleep induction in this medical circumstance appears to be mild. Our study settings analyzed patients who had no insomnia at home. The difficulty in falling asleep during PSG was mainly due to unfamiliar environment, discomfort caused by the PSG cables, or limitation of movement (ie, first night effect of PSG recordings). However, the subjective symptoms of sleep time and morning mood in suvorexant users were not worse compared with those at home in most patients, suggesting that suvorexant could have induced subjective natural sleep. In fact, suvorexant has been reported to improve sleep quality by reducing long wake bout times. ${ }^{25}$ Additionally, since several previous studies showed that suvorexant has less effects on sleep architecture and EEG power spectrum than other hypnotics, ${ }^{19,20}$ it would be better if patients during PSG can sleep well with suvorexant use. In the present setting, however, optimizing hypnotic use timing or the type of add-on hypnotics might not be enough to sleep well. Thus, further studies are needed to conclude the preferred use of suvorexant for patients during PSG.

Suvorexant is a dual orexin receptor antagonist. Orexin neurons originate in the lateral hypothalamus and project throughout the brain, including respiratory centers in the brain stem, ${ }^{26}$ suggesting the association of orexin and respiration. Nevertheless, suvorexant was not reported to increase respiratory events, possibly because an orexin receptor antagonist may have some effects on respiratory control during the awake state, but not during the sleep state. ${ }^{21}$ Suvorexant was reported not to affect respiratory events during sleep or oxygenation in patients with mildto-moderate OSA. ${ }^{27}$ In addition, suvorexant showed no consistent pattern of change of any sleep stages in healthy young men. ${ }^{28}$ Therefore, single-use suvorexant may have potential as a standard single-use hypnotic for some hospitalized patients with sleep onset insomnia during PSG. In addition, suvorexant might be preferable for patients with insomnia during out of center sleep testing at home. 
In this study, no adverse event was observed in all the patients. A large observational study of 53,081 patients in an European country revealed that $\mathrm{GABA}_{\mathrm{A}}$ receptor hypnotic agents (eg, lorazepam and zolpidem) were administered to $48.3 \%$ of hospitalized patients. ${ }^{6}$ In consideration of the potential side effects of hypnotic agents, $\mathrm{GABA}_{\mathrm{A}}$ receptor hypnotic agents might 1) modify sleep architecture, 2) increase respiratory events during sleep (cause respiratory depression), 3 ) increase the risk of falls (or bone fracture), and 4) cause

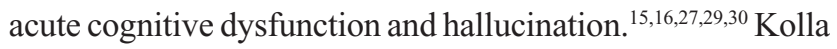
et $\mathrm{a}^{31}$ evaluated 36,141 patients undergoing PSG and reported adverse events in only $0.16 \%$, with falls being the second most frequent, at $0.05 \%$. No adverse events were observed in both patients taking suvorexant and those without hypnotics in this study. Nevertheless, residual daytime sleepiness was not evaluated in this study. Hence, since patients were discharged from the hospital soon after PSG, hypnotics with a short half-life should be more acceptable. Suvorexant has a relatively long half-life compared with short-acting $\mathrm{GABA}_{\mathrm{A}}$ hypnotics such as zolpidem. Hence, residual daytime sleepiness due to suvorexant use remains a concern. Five patients used antidepressants in the no insomnia group, and no patient used antidepressants in the insomnia group, with significant differences between the two groups. Four patients used selective serotonin reuptake inhibitor (SSRI), and one patient used both SSRI and serotonin noradrenaline reuptake inhibitor (SNRI). SSRI and SNRI have been reported to prolong sleep latency and worsen sleep continuity. ${ }^{32}$ Because the present study did not show any result concerning the influence of antidepressant agent, this effect remains unclear.

There were several limitations associated with this study. First, this study included a relatively small number of patients with OSA and it was conducted in a single hospital. Thus, the number of patients may have been insufficient to elucidate the actual incidence of adverse events because, in a previous report, falls were observed in only $0.8 \%$ of patients and hallucinations in only $0.4 \%$ among those prescribed $15 / 20 \mathrm{mg}$ of suvorexant in two 3-month phase III trials. ${ }^{23}$ Second, hypoventilation during PSG was not evaluated by $\mathrm{PaCO}_{2}$ monitoring. Accordingly, it was not possible to analyze the effect of suvorexant on ventilation, although such an effect of suvorexant has not been reported. Nonetheless, singleuse suvorexant had at least some effects on insomnia with sleep onset in patients with suspected OSA without obvious adverse effects. Third, we did not use a specific scale, such as the visual analog scale or a numeric rating scale, when analyzing patients' subjective evaluation of sleep during PSG. Therefore, our sleep questionnaire data could have been inaccurate. Fourth, the time of suvorexant administration was different in each patient, which could have affected sleep latency, sleep efficiency, and sleep questionnaire data. Fifth, zolpidem was administered to approximately half of the patients in the insomnia group. Zolpidem usage could have modified our results and worsened the results of the insomnia group because it was reported to decrease the minimum $\mathrm{SpO}_{2}$ and cause falls and delirium. ${ }^{33-35}$ However, because little is known about the efficacy (regarding both the effects on PSG and subjective sleep quality) and safety of single-use suvorexant during overnight PSG for patients with suspected OSA, the results of the present study may be useful for sleep clinicians and respiratory physicians.

\section{Conclusion}

In conclusion, suvorexant might have potential as a safe and effective (but mild) hypnotic during overnight PSG for patients with suspected OSA. However, further prospective studies are required to confirm the results of this study.

\section{Acknowledgments}

The authors sincerely thank Reiko Kunii and Shinobu Ikeda (Department of Laboratory Medicine, Chiba University Hospital) for the technical support of this research, and thank ENAGO (www.enago.jp) for the English language editing. This work was supported, in part, by a research grant to the Intractable Respiratory Diseases and Pulmonary Hypertension Research Group, the Ministry of Health, Labor and Welfare, the Japan Agency for the Medical Research and Development (AMED), and JSPS KAKENHI (Grant number 16K09528).

\section{Author contributions}

T Matsumura and J Terada performed the statistical analysis. All authors contributed to data analysis, conceptual design of the survey, drafting and revising the article, gave final approval of the version to be published, and agreed to be accountable for all aspects of the work.

\section{Disclosure}

The authors report no conflicts of interest in this work.

\section{References}

1. Newell J, Mairesse O, Verbanck P, Neu D. Is a one-night stay in the lab really enough to conclude? First-night effect and night-to-night variability in polysomnographic recordings among different clinical population samples. Psychiatry Res. 2012;200(2-3):795-801.

2. Agnew HW, Webb WB, Williams RL. The first night effect: an EEG study of sleep. Psychophysiology. 1966;2(3):263-266. 
3. Mccall C, Mccall WV. Objective vs. subjective measurements of sleep in depressed insomniacs: first night effect or reverse first night effect? J Clin Sleep Med. 2012;8(1):59-65.

4. Blackwell T, Paudel M, Redline S, Ancoli-Israel S, Stone KL; Osteoporotic Fractures in Men (MrOS) Study Group. A novel approach using actigraphy to quantify the level of disruption of sleep by in-home polysomnography: the MROS sleep study: sleep disruption by polysomnography. Sleep Med. 2017;32:97-104.

5. Curcio G, Ferrara M, Piergianni A, Fratello F, de Gennaro L. Paradoxes of the first-night effect: a quantitative analysis of antero-posterior EEG topography. Clin Neurophysiol. 2004;115(5):1178-1188.

6. Niedrig DF, Hoppe L, Mächler S, Russmann H, Russmann S. Benzodiazepine use during hospitalization: automated identification of potential medication errors and systematic assessment of preventable adverse events. PLoS One. 2016;11(10):e0163224.

7. Drummond GB. Comparison of sedation with midazolam and ketamine: effects on airway muscle activity. Br J Anaesth. 1996;76(5):663-667.

8. Gross JB, Smith L, Smith TC. Time course of ventilatory response to carbon dioxide after intravenous diazepam. Anesthesiology. 1982; 57(1):18-21.

9. Dolly FR, Block AJ. Effect of flurazepam on sleep-disordered breathing and nocturnal oxygen desaturation in asymptomatic subjects. Am J Med. 1982;73(2):239-243.

10. Höijer U, Hedner J, Ejnell H, Grunstein R, Odelberg E, Elam M. Nitrazepam in patients with sleep apnoea: a double-blind placebo-controlled study. Eur Respir J. 1994;7(11):2011-2015.

11. Cirignotta F, Mondini S, Zucconi M, Gerardi R, Farolfi A, Lugaresi E. Zolpidem-polysomnographic study of the effect of a new hypnotic drug in sleep apnea syndrome. Pharmacol Biochem Behav. 1988;29(4):807-809.

12. Marcantonio ER. Delirium in hospitalized older adults. $N$ Engl J Med 2017;377(15):1456-1466.

13. Mangusan RF, Hooper V, Denslow SA, Travis L. Outcomes associated with postoperative delirium after cardiac surgery. Am J Crit Care. 2015;24(2):156-163.

14. Neutel CI, Skurtveit S, Berg C. What is the point of guidelines? Benzodiazepine and z-hypnotic use by an elderly population. Sleep Med. 2012;13(7):893-897.

15. Dujardin K, Guieu JD, Leconte-Lambert C, Leconte P, Borderies P, de La Giclais B. Comparison of the effects of zolpidem and flunitrazepam on sleep structure and daytime cognitive functions. A study of untreated unsomniacs. Pharmacopsychiatry. 1998;31(1):14-18.

16. Brunner DP, Dijk DJ, Münch M, Borbély AA. Effect of zolpidem on sleep and sleep EEG spectra in healthy young men. Psychopharmacology. 1991;104(1):1-5.

17. Rhyne DN, Anderson SL. Suvorexant in insomnia: efficacy, safety and place in therapy. Ther Adv Drug Saf. 2015;6(5):189-195.

18. Sakurai T, Amemiya A, Ishii M, et al. Orexins and orexin receptors: a family of hypothalamic neuropeptides and $\mathrm{G}$ protein-coupled receptors that regulate feeding behavior. Cell. 1998;92(4):573-585.

19. Snyder E, Ma J, Svetnik V, et al. Effects of suvorexant on sleep architecture and power spectral profile in patients with insomnia: analysis of pooled phase 3 data. Sleep Med. 2016;19:93-100.
20. Ma J, Svetnik V, Snyder E, Lines C, Roth T, Herring WJ. Electroencephalographic power spectral density profile of the orexin receptor antagonist suvorexant in patients with primary insomnia and healthy subjects. Sleep. 2014;37(10):1609-1619.

21. Sun H, Palcza J, Rosenberg R, et al. Effects of suvorexant, an orexin receptor antagonist, on breathing during sleep in patients with chronic obstructive pulmonary disease. Respir Med. 2015;109(3):416-426.

22. Bennett T, Bray D, Neville MW, Suvorexant NMW. Suvorexant, a dual orexin receptor antagonist for the management of insomnia. $P T$. 2014;39(4):264-266.

23. Herring WJ, Connor KM, Ivgy-May N, et al. Suvorexant in patients with insomnia: results from two 3-month randomized controlled clinical trials. Biol Psychiatry. 2016;79(2):136-148.

24. Zucconi M, Ferri R, Allen R, et al. The official world association of sleep medicine (WASM) standards for recording and scoring periodic leg movements in sleep (PLMS) and wakefulness (PLMW) developed in collaboration with a task Force from the International Restless Legs Syndrome Study Group (IRLSSG). Sleep Med. 2006;7(2):175-183.

25. Svetnik V, Snyder ES, Tao P, et al. Insight into reduction of wakefulness by suvorexant in patients with insomnia: analysis of wake bouts. Sleep. 2018;41(1).

26. Peyron C, Tighe DK, van den Pol AN, et al. Neurons containing hypocretin (orexin) project to multiple neuronal systems. J Neurosci. 1998; 18(23):9996-10015.

27. Sun H, Palcza J, Card D, et al. Effects of Suvorexant, an Orexin Receptor Antagonist, on Respiration during Sleep In Patients with Obstructive Sleep Apnea. J Clin Sleep Med. 2016;12(1):9-17.

28. Sun H, Kennedy WP, Wilbraham D, et al. Effects of suvorexant, an orexin receptor antagonist, on sleep parameters as measured by polysomnography in healthy men. Sleep. 2013;36(2):259-267.

29. Brandt J, Leong C. Benzodiazepines and Z-Drugs: an updated review of major adverse outcomes reported on in epidemiologic research. Drugs in $R \& D .2017 ; 17(4): 493-507$.

30. Wong CK, Marshall NS, Grunstein RR, et al. Spontaneous adverse event reports associated with zolpidem in the United States 2003-2012. J Clin Sleep Med. 2017;13(2):223-234.

31. Kolla BP, Lam E, Olson E, Morgenthaler T. Patient safety incidents during overnight polysomnography: a five-year observational cohort study. J Clin Sleep Med. 2013;9(11):1201-1205.

32. Schweitzer PK, Griffin KS. Medication effects on sleep. In: Avidan A, editor. Review of Sleep Medicine 4th ed. Philadelphia: Elsevier; 2017:406.

33. Drake CL, Durrence H, Cheng P, et al. Arousability and fall risk during forced awakenings from nocturnal sleep among healthy males following administration of zolpidem $10 \mathrm{mg}$ and doxepin $6 \mathrm{mg}$ : a randomized, placebo-controlled, four-way crossover trial. Sleep. 2017;40(7).

34. Kolla BP, Lovely JK, Mansukhani MP, Morgenthaler TI. Zolpidem is independently associated with increased risk of inpatient falls. J Hosp Med. 2013;8(1):1-6.

35. Mason M, Cates CJ, Smith I. Effects of opioid, hypnotic and sedating medications on sleep-disordered breathing in adults with obstructive sleep apnoea. Cochrane Database Syst Rev. 2015;7:Cd011090. 


\section{Supplementary material}

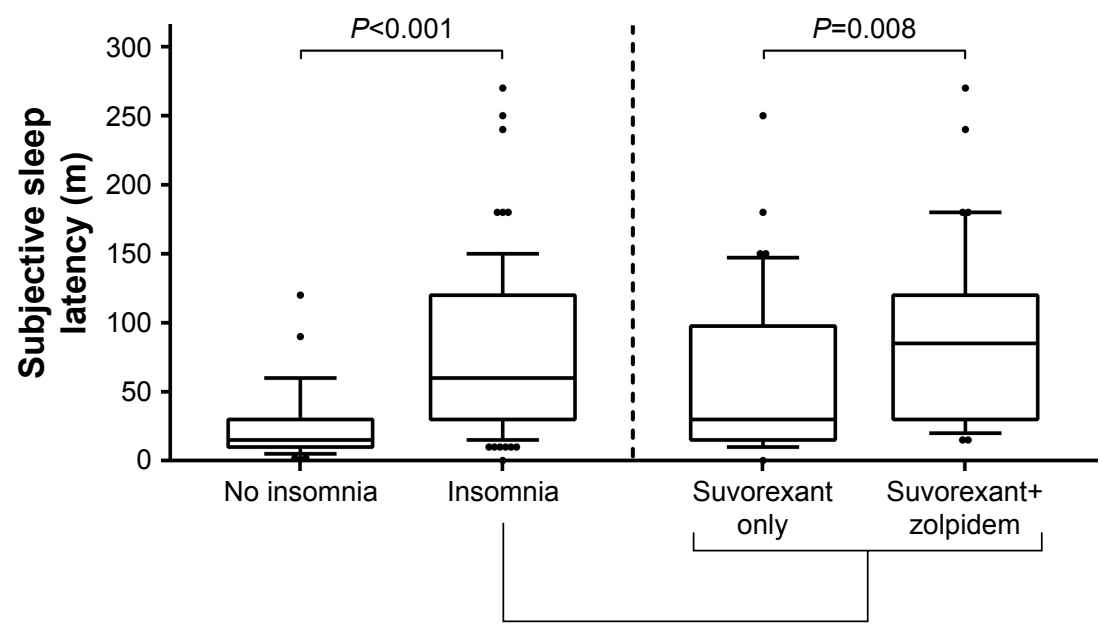

Figure SI Summary in the questionnaire of subjective sleep latency.

Notes: The subjective sleep latency was significantly longer in the insomnia group compared to the no insomnia group. Among the insomnia group, the subjective sleep latency in the no insomnia group was significant shorter compared to the insomnia group. Furthermore, subjective sleep latency was significantly shorter in the suvorexant only group than in the suvorexant+zolpidem group.

\section{Publish your work in this journal}

Drug Design, Development and Therapy is an international, peerreviewed open-access journal that spans the spectrum of drug design and development through to clinical applications. Clinical outcomes, patient safety, and programs for the development and effective, safe, and sustained use of medicines are the features of the journal, which

has also been accepted for indexing on PubMed Central. The manuscript management system is completely online and includes a very quick and fair peer-review system, which is all easy to use. Visit http://www.dovepress.com/testimonials.php to read real quotes from published authors. 